Research Article

\title{
Critical Incident Disclosing Behaviors and Associated Factors among Nurses Working in Amhara Region Referral Hospitals, Northwest Ethiopia: A Cross-Sectional Study
}

\author{
Yeshiambew Eshete, ${ }^{1}$ Bekele Tesfaye, ${ }^{2}$ Zewdu Dagnew, ${ }^{2}$ Demewoz Kefale, ${ }^{1}$ \\ Demke Mesfin Belay, ${ }^{1}$ and Binyam Minuye ${ }^{1}{ }^{1}$ \\ ${ }^{1}$ Department of Nursing, College of Health Sciences, Debretabor University, Debretabor, Ethiopia \\ ${ }^{2}$ College of Health Sciences, Debremarkos University, Debremarkos, Ethiopia \\ Correspondence should be addressed to Binyam Minuye; biniamminuye@yahoo.com
}

Received 22 August 2020; Revised 1 December 2020; Accepted 31 December 2020; Published 12 January 2021

Academic Editor: Fred A. Luchette

Copyright (c) 2021 Yeshiambew Eshete et al. This is an open access article distributed under the Creative Commons Attribution License, which permits unrestricted use, distribution, and reproduction in any medium, provided the original work is properly cited.

\begin{abstract}
Background. Though the goal of healthcare institutions is patient safety, errors have been committed by healthcare providers. Incident reporting behavior enhances patient safety by reducing the repeated occurrence of errors in the health facility. Therefore, this study aims to identify incident disclosing behaviors and associated factors among nurses working in referral hospitals, Northwest Ethiopia. Methods. Institution-based cross-sectional study design was conducted among randomly selected 319 nurses working in referral hospitals of Amhara region from March 1-30, 2019. Data were collected using a self-administered structured questionnaire. Data were coded and entered into EpiData 4.2 software and exported to Statistical Package for Social Sciences version 25 for analysis. All variables with $p$ value $<0.25$ during bivariable binary logistic regression analysis were considered for multivariable binary logistic regression analysis. Odds ratio along with $95 \%$ CI was estimated to measure the strength of the association. Level of statistical significance was declared at $p$ value $\leq 0.05$. Results. The proportion of nurses who reported incidents was $31.9 \%$ ( $95 \%$ confidence interval (CI), 27, 3)). Fear of administrative sanctions (adjusted odd ratio $(\mathrm{AOR})=0.45 ; 95 \% \mathrm{CI}, 0.22,0.90$ ), fear of legal penalty (AOR $=0.27 ; 95 \% \mathrm{CI}, 0.14,0.50)$, lack of feedback $(\mathrm{AOR}=0.29 ; 95 \% \mathrm{CI}, 0.13,0.66)$, nonsupportive environment $(\mathrm{AOR}=0.27 ; 95 \% \mathrm{CI}, 0.14,0.52)$, and feel that reporting to colleague is easier $(\mathrm{AOR}=2.65 ; 95 \% \mathrm{CI}, 1.35,5.20)$ were all found to be significant factors. Conclusions. The proportion of nurses who reported incidents was low. Fear of administrative sanctions, fear of legal penalty, lack of feedback, nonsupportive environment, and felling that reporting to colleagues was easier are found to be significant factors. Developing a system that encourages critical incident reporting behavior and provide protection from penalties for nurses to report incidents for the enhancement of patient safety and quality of care at each health facility and regional level is crucial.
\end{abstract}

\section{Background}

Incidence is an undesirable adverse event, injury, and a near miss that lead to patient's harm. Errors can occur in all stages in the process of care delivery [1-3]. Incident reporting is a tool that enables nurses to disclose incidents to others such as colleagues, patients, and concerned bodies (Administrative personnel's, supervisors, and directors). Incident reporting permits a retrospective window on the healthcare system by providing extremely valuable information for prevention of future errors, by identifying weaknesses of the system and detecting preventable events $[4,5]$; however, many of the incidents are not reported.

Medical error is an inevitable and continuous problem in healthcare facilities. It increases from time to time and poses a great threat to the safety of patients and quality of care [2, 6]. According to 1999 Institute of Medicine (IOM) report, between 44,000 and 98,000 people die annually as a result of medical errors, and studies in the United States of America also estimated that the number of people died due to medical incident accounts 142,000 [7-9]. 
To minimize and avoid the repeated occurrence of incidents, healthcare facilities need to change policies and procedures that encourage nurses to report incidents. In addition, the protocol also creates an opportunity to enhance nurses to learn from mistakes, and this will also further enhance patient safety $[10,11]$. Studies recommend that reoccurrence of errors can be prevented by the incident reporting system. However, lack of reporting of incidents by nurses as well as health facility leaders increases incidents in an alarming rate which affects patient safety throughout the world $[12,13]$.

Identification, documentation, and analyzing of actual or potential harm to patients as well as timely report is crucial for the development and functioning of safer healthcare systems which require combined changes of healthcare facility leaders and healthcare workers $[14,15]$. Despite the significance of contribution of incident reporting to patient safety and quality of care, underreporting behaviors of nurses due to different factors such as sanction, deployment, and penalty are high. There are evidences regarding the burden of incident in developed countries, but little is known in developing countries such as Ethiopia. Therefore, the current study was intended to fill the information gap on incident reporting behavior of nurses working in Amhara region referral hospitals.

\section{Methods}

2.1. Study Design, Period, Setting, and Population. An institution-based cross-sectional study was conducted from March 1-30, 2019, at referral hospitals of Amhara region (Debre Markos Referral Hospital, Debre Birhan Referral Hospital, Dessie Referral Hospital, and Felege Hiwot Referral Hospital), Ethiopia. All nurses working in referral hospitals of Amhara regional state with $\geq 6$ month experience were the target population. Those nurses who were giving free service and annual leave were excluded. Finally, 319 nurses were included in the study.

2.2. Data Collection Methods. Data were collected by using a structured self-administered English version questionnaire which was adapted from a study conducted by Engeda [10] with the authors' permission. The tool was composed of four parts, such as sociodemographic characteristics of nurses, levels of incident reporting, institutional factors, and perceived barriers of nurses to incident reporting. The data were collected by four nurses, two Bachelor of Science degree (BSc) nurse supervisors. Completeness of each recording format was checked before collecting the data.

\subsection{Variables}

2.3.1. Dependent Variable. Incident disclosing behavior is the dependent variable.

\subsubsection{Independent Variables}

(i) Sociodemographic characteristics: age, sex, nursing educational level, years of service as a nurse, working hours per week, and primary work area (ii) Institutional factors: training on incident reporting, availability of guideline/policy, and availability of reporting formats

(iii) Perceived barriers: nonsupportive environment (culture of shame and blame), loss of prestige among colleagues, fear of legal or financial penalties, fear of administrative sanction, and lack of feedback

\subsection{Operational Definitions}

(i) Incident reporting behavior: nurses' action towards the error they encounter during the delivery of care to report or not report (disclose) to their colleague or concerned body $[10,16]$

(ii) High incident reporting behavior: nurses who choose the option "always" for each item of outcome measuring questions listed and score greater or equal to the mean [5]

(iii) Low incident reporting behavior: nurses who choose one of the options "most of the time," "sometimes," "rarely," or "not reported at all" for each item of outcome measuring questions listed above and score below to the mean [10]

2.5. Data Quality Control. The pretest was performed on $5 \%$ of the sample size one week before the actual data collection time. One day training was given for data collectors and supervisors on data collection tools and data collection procedures. Completeness of each data collection tool had been checked by the principal investigator and the supervisors in a daily base. Double data entry was performed by two data clerks, and the consistency of the entered data was cross-checked.

2.6. Data Processing and Analysis. The data were entered, coded, cleaned, and checked using EpiData statistical software version 4.2.0.0, and analysis was performed by using SPSS version 25 statistical software. Summation of the scores for the scale incident reporting behavior was made to determine the level of incident reporting. The data with the Likert scale were analyzed as categorical data (the scores for three outcome variables were summed up and then categorized). Descriptive statistics such as proportions, percentages, ratios, frequency distributions, and appropriate graphical presentations were used for describing the data. All variables with a $p$ value $\leq 0.25$ in the bivariable binary logistic regression analysis were entered into the final model for multivariable analysis. Variables with $p$ values $\leq 0.05$ were considered as statistically significant with the outcome variable.

2.7. Ethical Considerations. Ethical clearance was obtained from Debre Markos University, College of Health Sciences, and Institutional Health Research Ethics Review Committee (IHRERC). Then, official letters were written to each referral hospital for permission and support. Permission letter was obtained from the selected hospitals. Written consent was 
TABLE 1: Sociodemographic characteristics of nurses working in Amhara regional state referral hospitals, $2019(n=288)$.

\begin{tabular}{|c|c|c|}
\hline Variable & Frequency & Percentage \\
\hline \multicolumn{3}{|l|}{ Age } \\
\hline 20-29 years & 168 & 58.3 \\
\hline $30-39$ years & 87 & 30.2 \\
\hline 40-49 years & 25 & 8.7 \\
\hline $50-60$ years & 8 & 2.8 \\
\hline \multicolumn{3}{|l|}{ Sex } \\
\hline Male & 164 & 56.9 \\
\hline Female & 124 & 43.1 \\
\hline \multicolumn{3}{|l|}{ Marital status } \\
\hline Single & 103 & 35.8 \\
\hline Married & 185 & 64.2 \\
\hline \multicolumn{3}{|l|}{ Religion of participant } \\
\hline Orthodox Christian & 245 & 85.1 \\
\hline Muslim & 39 & 13.5 \\
\hline Protestant & 2 & 1.4 \\
\hline \multicolumn{3}{|l|}{ Ethnicity of participant } \\
\hline Amhara & 276 & 95.8 \\
\hline Others & 12 & 4.2 \\
\hline \multicolumn{3}{|l|}{ Educational level } \\
\hline Diploma nurse & 27 & 9.4 \\
\hline BSc nurse & 253 & 87.8 \\
\hline MSc nurse & 8 & 2.8 \\
\hline \multicolumn{3}{|l|}{ Position of nurse } \\
\hline Head nurse & 24 & 8.3 \\
\hline Staff nurse & 264 & 91.7 \\
\hline \multicolumn{3}{|l|}{ Year of service as nurse } \\
\hline $1-5$ & 146 & 50.7 \\
\hline $6-10$ & 91 & 31.6 \\
\hline $11-15$ & 23 & 8 \\
\hline $16-20$ & 18 & 6.3 \\
\hline 21 and above & 10 & 3.4 \\
\hline \multicolumn{3}{|l|}{ Working hours per week } \\
\hline 39-49 hours & 73 & 25.4 \\
\hline $50-60$ hours & 170 & 59 \\
\hline Above 60 hours & 45 & 15.6 \\
\hline
\end{tabular}

taken from each participant, and confidentiality was assured. The data collected was used for the study purpose. Privacy and anonymity was secured.

\section{Results}

3.1. Sociodemographic Characteristics of the Study Participants. In this study, a total of 288 nurses were participated in the study making a response rate of $90.3 \%$, of which $56 \%$ (162) was male. The median age of nurses was about 28.5 with an interquartile range (IQR) of 26.5-32 years. Majority, 245 (85\%) nurses were orthodox Christian. Almost all 276 (96\%) were Amhara in ethnicity. The mean working hours per week was 54.07 ( \pm 9.84$)$ (Table 1).

3.2. Incident Disclosing Behavior. A total of 288 nurses participated in the study, and the finding showed that overall incidents reporting behavior of nurses was $31.9 \%$ (95\% CI, 27-37).
3.3. Factor Associated with Incident Reporting Behavior. A binary logistic regression was performed to identify the association between incident reporting behavior and independent variables. In bivariable logistic regression, age, sex, taking, training on incident reporting, availability of the reporting format, fear of administrative sanctions, fear of financial or legal penalty, lack of feedback, reporting to senior nurses, reporting to colleagues, reporting to medical director, working hours per week, develop a system to minimize repetition of error, religion, and nonsupporting (culture of shame or blame) environment met the criteria to be fitted into a multivariable analysis ( $p$ value $\leq 0.25$ ).

Fear of administrative sanctions $(\mathrm{AOR}=0.45,95 \% \mathrm{CI}$, $0.22,0.90)$, fear of legal penalties $(\mathrm{AOR}=0.27,95 \% \mathrm{CI}, 0.14$, 0.500 ), nonsupporting environment (culture of shame or blame $)(\mathrm{AOR}=0.27,95 \% \mathrm{CI}, 0.14,0.52)$, lack of feedback $(\mathrm{AOR}=0.29,95 \% \mathrm{CI}, 0.13,0.66)$, and reporting to colleague is easier $(\mathrm{AOR}=2.65,95 \% \mathrm{CI}, 1.35,5.20)$ were significantly associated with the outcome variable in multivariable analysis. Accordingly, nurses who feared administrative sanctions were 55\% less likely to report incident as compared to those nurses who did not fear administrative sanctions. Beside, nurses who feared legal penalty and working in nonsupporting environment were $73 \%$ less likely to report incidents as compared to its counterpart. Moreover, nurses who believed that reporting to colleague is easier were 2.65 times more likely to report the incident than those nurses who did not believe reporting to colleague. Finally, providing feedback for nurse's increases incident reporting behavior of nurses by $61 \%$ (Table 2 ).

\section{Discussion}

Incident reporting systems in hospitals provide valuable data and information for improving patient safety through analysis of the nature and patterns of incidents that have been occurred; it can be used for learning and grounds for precautionary action in the future [14]. This study showed the incident reporting behavior and associated factors in referral hospitals.

The overall incident reporting behavior was 31.9\% (95\% CI, 27-37) which is higher than the study conducted in Gondar Comprehensive Specialized Hospital (25.4\%) [10]. The difference between the study setting and period was contributed for the discrepancy. According to the Ethiopian hospital reform implementation guideline, an incident officer is assigned to each hospital to receive and investigate all incident reports, and a summary report of all incidents must be submitted to a quality assurance committee of each hospital [17].

But lower than the study conducted in Egypt (66.7\%), Korea (53\%), and Iran $(50.26 \%)$ [3, 18, 19]. The reason for this discrepancy may be accounted for legal protection of reporters, blame for the reported errors in the working environment, and lack of personal confidence to withstand any consequence of error reporting. The result of a study from Iran showed that the reasons that nurses do not report incidents were fear, disagreement over the occurred mistake, 
TABLE 2: Bivariable and multivariable analysis of incident reporting behaviors among nurses working in Amhara regional state referral hospitals, $2019(n=288)$.

\begin{tabular}{|c|c|c|c|c|}
\hline \multirow{2}{*}{ Variable } & \multicolumn{2}{|c|}{$\begin{array}{l}\text { Incident } \\
\text { reporting }\end{array}$} & \multicolumn{2}{|c|}{ Odds ratio $(95 \% \mathrm{CI})$} \\
\hline & Yes & No & COR & AOR \\
\hline \multicolumn{5}{|c|}{ To whom reporting is easier to colleagues } \\
\hline Yes & 30 & 29 & $2.79(1.55-5.02)$ & $2.65(1.35-5.20)^{*}$ \\
\hline No & 62 & 167 & 1 & 1 \\
\hline \multicolumn{5}{|c|}{ Reporting to senior nurse } \\
\hline Yes & 27 & 85 & $0.54(0.22-0.92)$ & $0.71(0.48,2.88)$ \\
\hline No & 65 & 111 & 1 & \\
\hline \multicolumn{5}{|c|}{ Reason to report to develop a system } \\
\hline Yes & 24 & 87 & $0.44(0.26-0.76)$ & $0.32(0.12,1.45)$ \\
\hline No & 68 & 109 & 1 & \\
\hline \multicolumn{5}{|c|}{ Organizational factors training on error reporting } \\
\hline Trained & 23 & 107 & $0.28(0.16-0.48)$ & $0.67(0.23,1.20)$ \\
\hline Not trained & 69 & 89 & 1 & \\
\hline \multicolumn{5}{|c|}{ Availability of report format } \\
\hline Yes & 25 & 38 & $1.55(0.87-2.77)$ & $1.34(0.67,2.8)$ \\
\hline No & 67 & 158 & 1 & 1 \\
\hline \multicolumn{5}{|c|}{ Perceived barrier to report nonsupportive environment } \\
\hline Yes & 20 & 97 & $0.28(0.16-0.50)$ & $0.27(0.14-0.52)^{*}$ \\
\hline No & 72 & 99 & 1 & 1 \\
\hline \multicolumn{5}{|c|}{ Fear of administrative sanction } \\
\hline Yes & 16 & 86 & $0.27(0.15-0.49)$ & $0.45(0.22-0.90)^{*}$ \\
\hline No & 76 & 110 & 1 & 1 \\
\hline \multicolumn{5}{|c|}{ Fear of legal penalty } \\
\hline Yes & 24 & 116 & $0.24(0.14-0.42)$ & $0.27(0.14-0.50)^{*}$ \\
\hline No & 68 & 80 & 1 & 1 \\
\hline \multicolumn{5}{|c|}{ Lack of feedback } \\
\hline Yes & 12 & 47 & $0.48(0.24-0.95)$ & $0.29(0.13-0.66)^{*}$ \\
\hline No & 80 & 149 & 1 & 1 \\
\hline
\end{tabular}

${ }^{*} p$ value $<0.05$.

administrative reactions to errors, and the effort needed for the reporting process [20].

Similarly, this study revealed that nurses who believed that there is lack of feedback after reporting were $71 \%$ less likely to report incidents than its counterpart which is in line with studies conducted in the USA and Turkey [21,22]. This could be due to the fact that nurses believed in a lack of root cause analysis, which is used as a means to understand factors contributing to medical errors and identify systemic factors that contribute to errors. It is the action or response to the reporting that brings the change within an organization. Reporting of incidents should lead to an in-depth investigation to assess the etiological factors. Thus, incident reporting without a response from the concerned body leads to a sense of futility in the incident reporting period.

Nurses who feared legal penalties were $73 \%$ less likely to report incidents than those who did not fear legal penalties. This is consistent with the result of studies conducted in Egypt and Iran $[3,20]$. This fear might be due to adverse consequences of reporting such as a malpractice lawsuit, losing patients' trust, emotional reactions of the patients and their relatives, or losing occupational position. An incident that harmed or did not harm the patient also negatively influences the reporting behavior of nurses. The result of the study showed that most of nurses reported that an incident report could be used against them and there would not be legal protection. Besides, the penal code of Ethiopia state that hurting a patient as a result of negligence or medical error is punitive even if it is unintentional [10].

This finding also showed that nurses who believed there is a nonsupportive environment (a culture of blame and shame) were $73 \%$ less likely to report incidents than those believed they worked in a supportive environment. This is in agreement with results of studies conducted in Saudi Arabia and Korea $[23,24]$ which stated that nonsupportive environment prevents nurses from reporting incidents. This could be attributed to the cultural practice of naming and shaming that involved in medical errors reporting during meetings by facility managers and head nurses. A nurse who reports errors is considered as incompetent by communities of the institutions. As a result of this environment, nurses favor not reporting than disclosing the mistake to the concerned body.

Nurses who feared administrative sanctions were 55\% less likely to report incidents than those who did not fear administrative sanctions. This finding is in line with previous studies conducted in Ethiopia and Iran [10, 20]. This could be attributed to the fact that nurses feel insecure about their job and are afraid that they will have to face administrative sanctions such as economic and disciplinary measures that could be taken after committing and reporting an error and health facility administration's focus on the person who 
committed errors rather than a system in which medical errors can be registered and analyzed [19]. In the context of Ethiopia, job profile quality has a great value for future nurse's career but reporting of errors results in oral or written warning which is attached to their files, and this ends up demotion to dismissal from the organization. Due to those consequences, nurses try to hold the incident from reporting unless there is a system that protects them from such kind of administrative interference.

Nurses who believed that reporting to colleague is easy were 2.65 times more likely to report incidents than those who did not believe that reporting to a colleague is not easy. The possible reason could be colleagues are easily found in the nearby as well as need them to control the adverse effects of mistakes committed by nurses. On top of that, the consequences of medical errors are overwhelming and psychologically unhealthy for the practicing nurses as well. Therefore, colleague is the preferred one to obtain the solution for escaping from this tension and keeping the occurrence of incident confidentially.

4.1. Limitation of the Study. The study participants were only nurses and social desirability bias.

\section{Conclusions}

The proportion of nurses who report all type of incidents in all situations is low. But, improved reporting behavior was demonstrated when the incident is caught and corrected before affecting the patient. The study also found important factors that prevent them from reporting errors such as nonsupportive environment, fear of administrative sanction, fear of legal penalty, lack of feedback, and reporting to colleagues is easier.

\section{Abbreviations}

AOR: Adjusted odds ratio

DBRH: Debre Birhan Referral Hospital

DMRH: Debre Markos Referral Hospital

DRH: Dessie Referral Hospital

FHCSH: Felege Hiwot comprehensive specialized hospital

IRS: Incident reporting system

IOM: Institute of Medicine

ME: $\quad$ Medical error

SD: $\quad$ Standard error

WHO: World Health Organization.

\section{Data Availability}

All relevant data are included within the article and are available from the corresponding author upon request.

\section{Ethical Approval}

Ethical clearance was obtained from Debre Markos University, College of Health Sciences, and Institutional Health Research Ethics Review Committee (IHRERC).

\section{Conflicts of Interest}

The authors declare that they have no conflicts of interest.

\section{Authors' Contributions}

YE, BT, and ZD worked on designing the study, training the data collectors, supervising the data collectors, and interpreting the result. $\mathrm{BM}, \mathrm{DK}$, and DM analyzed the result and wrote the manuscript. All authors involved starting from design, data interpretation, and to critically review the manuscript.

\section{Acknowledgments}

The authors acknowledge nurses who participated in the study and data collectors.

\section{References}

[1] A. Gatasheh, J. Alkhawaldeh, and D. B. Hani, "Documentation of incident reports by nurses in Jordanian accredited private hospitals: types and causes a systematic review," IOSR Journal of Nursing and Health Science, vol. 6, no. 2, pp. 101-106, 2017.

[2] L. L. Pietra, L. Calligaris, L. Molendini, R. Quattrin, and S. Brusaferro, "Medical errors and clinical risk management: state of the art," Acta Otorhinolaryngologica Italica, vol. 25, no. 6, pp. 339-346, 2005.

[3] E. Araby, R. Eldesouky, and H. Abed, "Medical errors among nurses in the university hospital of Benha, Egypt: forms, underlying factors and reporting," Biomedical Journal of Scientific \& Technical Research, vol. 7, no. 4, 2018.

[4] S. M. Evans, B. J. Smith, A. Esterman et al., "Evaluation of an intervention aimed at improving voluntary incident reporting in hospitals," Quality and Safety in Health Care, vol. 16, no. 3, pp. 169-175, 2007.

[5] A. Jember, M. Hailu, A. Messele et al., "Proportion of medication error reporting and associated factors among nurses: a cross sectional study," BMC Nursing, vol. 17, no. 9, pp. 1-8, 2016.

[6] J. Y. Park, "The current state of medical error in south Korea," Scholar Journal of Applied Sciences and Research, vol. 1, no. 4, pp. 28-33, 2018.

[7] G. U. P. Iloh, A. Chuku, and A. N. Amadi, "Medical errors in Nigeria: a cross-sectional study of medical practitioners in Abia state," Archives of Medicine and Health Sciences, vol. 5, pp. 44-49, 2017.

[8] M. A. Makary and M. Daniel, "Medical error-the third leading cause of death in the US," BMJ Open, vol. 353, pp. 1-5, 2016.

[9] M. C. Balas, L. D. Scott, and A. E. Rogers, "The prevalence and nature of errors and near errors reported by hospital staff nurses," Applied Nursing Research, vol. 17, no. 4, pp. 224-230, 2004.

[10] E. H. Engeda, "Incident reporting behaviours and associated factors among nurses working in Gondar university comprehensive specialized hospital, northwest Ethiopia," Scientifica, vol. 2016, Article ID 6748301, 7 pages, 2016.

[11] M. A. Banakhar, A. I. Tambosi, S. Al-Ameen et al., "Barriers of reporting errors among nurses in a tertiary hospital," International Journal of Nursing \& Clinical Practices, vol. 4, pp. 1-7, 2017. 
[12] H. Safarpour, M. Tofighi, L. Malekyan, J. Bazyar, S. Varasteh, and R. Anvary, "Patient safety attitudes, skills, knowledge and barriers related to reporting medical errors by nursing students," International Journal of Clinical Medicine, vol. 8, no. 1, pp. 1-11, 2017.

[13] T. Hossain and N. Hossain, "Incident reporting in surgery: a review of the literature," International Surgery Journal, vol. 2, no. 2, pp. 157-160, 2015.

[14] M. Garrouste-Orgeas, F. Philippart, C. Bruel et al., "Overview of medical errors and adverse events," Annals of Intensive Care, vol. 2, no. 2, pp. 1-9, 2012.

[15] L. Cloete, "Reducing medication errors in nursing practice," Nursing Standard, vol. 29, no. 20, pp. 50-59, 2015.

[16] A. Y. Alam and A. S. Mir, "Attitudes and perceived barriers of tertiary level health professionals towards incident reporting in Pakistan," North American Journal of Medical Sciences, vol. 2, no. 2, pp. 100-105, 2010.

[17] Ministry of Health, Ethiopian Hospital Reform Implementation Guideline, Ministry of Health, Ethiopia, 2010.

[18] J.-I. Hwang and J. Ahn, "Teamwork and clinical error reporting among nurses in Korean hospitals," Asian Nursing Research, vol. 9, no. 1, pp. 14-20, 2015.

[19] J. Poorolajal, S. Rezaie, and N. Aghighi, "Barriers to medical error reporting," International Journal of Preventive Medicine, vol. 6, p. 97, 2015.

[20] H. Peyrovi, A. Nikbakht Nasrabadi, and S. Valiee, "Exploration of the barriers of reporting nursing errors in intensive care units: a qualitative study," Journal of the Intensive Care Society, vol. 17, no. 3, pp. 215-221, 2016.

[21] J. C. Pham, T. Girard, and P. J. Pronovost, "What to do with healthcare incident reporting systems," Journal of Public Health Research, vol. 2, no. 27, pp. 154-158, 2013.

[22] A. Ünal and S. Seren, "Medical error reporting attitudes of healthcare personnel, barriers and solutions: a literature review," Journal of Nursing \& Care, vol. 5, no. 6, pp. 1-8, 2016.

[23] A. M. S. Alduais, S. Mogali, B. Al Shabrain et al., "Barriers and strategies of reporting medical errors in public hospitals in Riyadh city: a survey-study," IOSR Journal of Nursing and Health Science, vol. 3, no. 5, pp. 72-85, 2014.

[24] J.-I. Hwang, S.-I. Lee, and H.-A. Park, "Barriers to the operation of patient safety incident reporting systems in Korean general hospitals," Healthcare Informatics Research, vol. 18, no. 4, pp. 279-285, 2012. 